\title{
$N$-Ac-Leukotriene $\mathrm{E}_{\mathbf{4}}$ \\ Unique Vascular Activity in the Conscious Rat
}

\author{
G. FEUERSTEIN, ${ }^{a}$ G. LETTS, ${ }^{b}$ AND A-L. SIREN ${ }^{a}$ \\ ${ }^{a}$ Department of Neurology \\ Uniformed Services University of the Health Sciences \\ Bethesda, Maryland 20814 \\ ${ }^{b}$ Merck Frosst \\ Dorval, Canada H9R $4 P 8$
}

$N$-Acetyl-leukotriene $\left.\mathrm{E}_{4}(N \text {-Ac-LTE })_{4}\right)$ is a recently discovered metabolite of the cysteinyl leukotrienes (LT). ${ }^{l}$ Although $N$-Ac-LTE ${ }_{4}$ has been identified so far only in the bile, an enterohepatic cycle for LT has been suggested ${ }^{2}$ that might provide for some LT metabolites access to the systemic circulation. The biological significance of

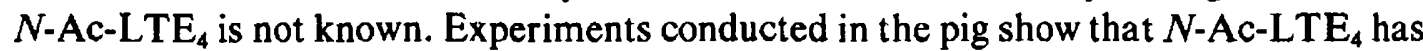
very weak actions on the cardiovascular and respiratory systems. ${ }^{3}$ However, the formation of $N$-Ac-LTE 4 has not been shown as yet in the pig. Therefore, we have decided to examine the effect of $N$-Ac-LTE $\mathrm{L}_{4}$ in the rat, where $\mathrm{LTC} / \mathrm{D}_{4} / \mathrm{E}_{4}$ have potent cardiovascular effects and $N-\mathrm{Ac}_{-} \mathrm{LTE}_{4}$ is a primary metabolite of the LT.

Rats were anesthetized with ketamine-acepromazine. Next, a Doppler flow probe was implanted on the renal (R), mesenteric (M), and abdominal aorta (for hindquarter blood flow, HQ), and a PE-50 catheter was placed in the femoral artery for blood pressure (BP) monitoring. $N$-Ac-LTE 4 (Merck Frosst) was administered to the

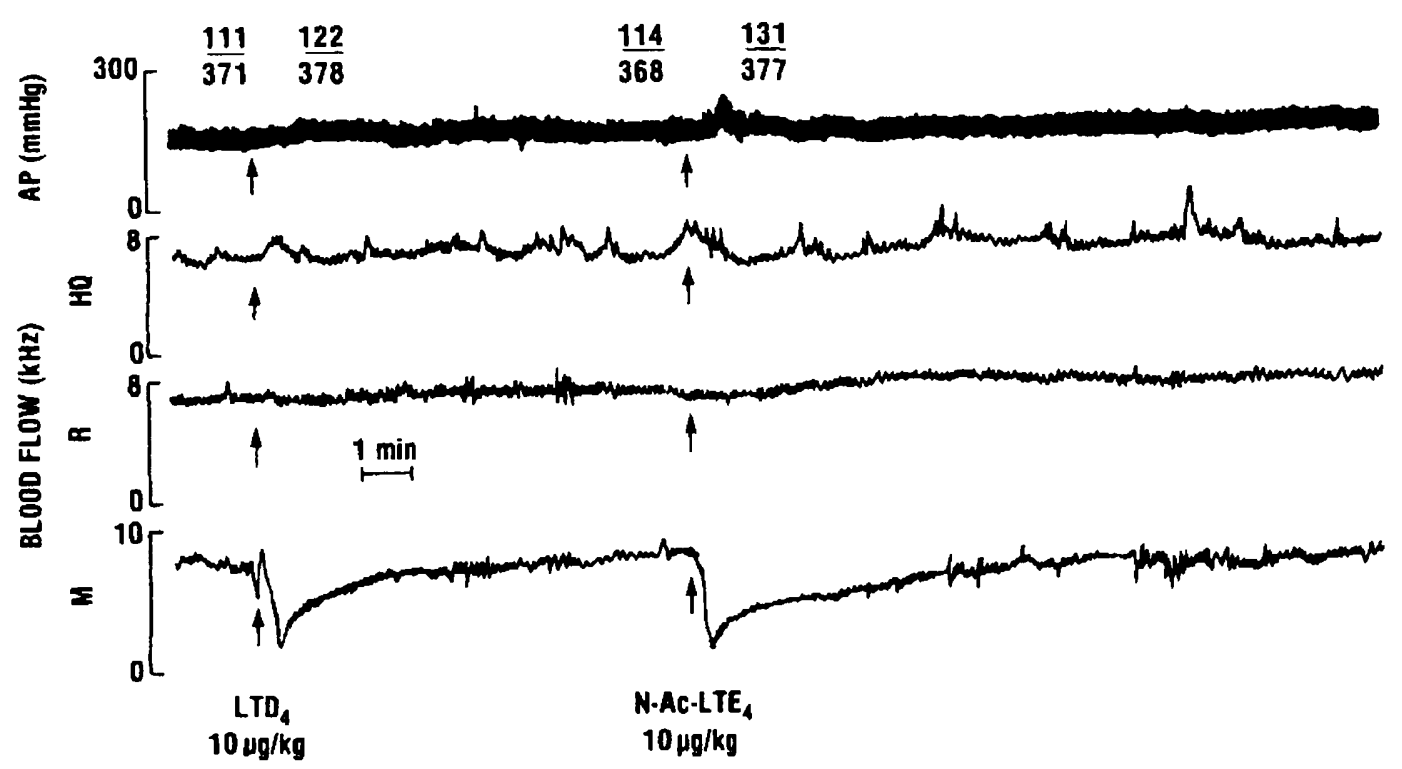

FIGURE 1. Authentic recording of the cardiovascular effects of $N$-Ac-LTE ${ }_{4}$ in the conscious rat. $\mathrm{AP}$ = arterial pressure. $\mathrm{M}, \mathrm{R}$, and $\mathrm{HQ}$ represent the superior mesenteric, renal, and hindquarter

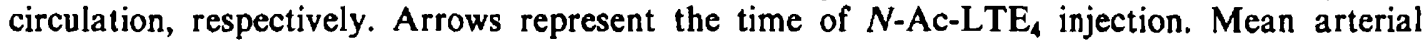
pressure and heart rate are given as numerator/denominator values, respectively. 
conscious rat $(0.1-30 \mu \mathrm{g} / \mathrm{kg})$ and blood flow $(\mathrm{BF})$ in the $\mathrm{R}, \mathrm{M}$, and HQ regions was continuously recorded, as was the systemic $\mathrm{BP}$ and heart rate.

$N$-Ac-LTE $4(n=6)$ produced only a moderate pressor response $(5-15 \mathrm{mmHg})$. However, MBF was reduced in a dose-dependent manner (up to $-50 \%$ at $30 \mu \mathrm{g} / \mathrm{kg}$ ), along with an increase in the MVR: $300 \%(30 \mu \mathrm{g} / \mathrm{kg}) . N-\mathrm{Ac}^{-L_{T E}} \mathrm{E}_{4}$ had no effect at all on the renal or HQ circulation in the dose range studied (FIGURE 1). Comparison of the duration of the effect of $N$-Ac-LTE 4 on MBF to that of $\mathrm{LTD}_{4}$ revealed a more prolonged effect of $\mathrm{N}$-Ac-LTE 4 (FIGURE 2).

The data presented in this report clearly show that $N$-Ac-LTE $\mathrm{LE}_{4}$ is a biologically active metabolite of LT. $N$-Ac-LTE 4 had a preferential effect on the mesenteric circulation with virtually no effect on the $R$ and $H Q$ circulation. In this regard, $N$-Ac-LTE $\mathrm{L}_{4}$ is more specific than $\mathrm{LTD}_{4}$ or $\mathrm{LTE}_{4}$, which produce significant renal and HQ vasoconstriction in comparable doses ${ }^{4}$ in this same model.

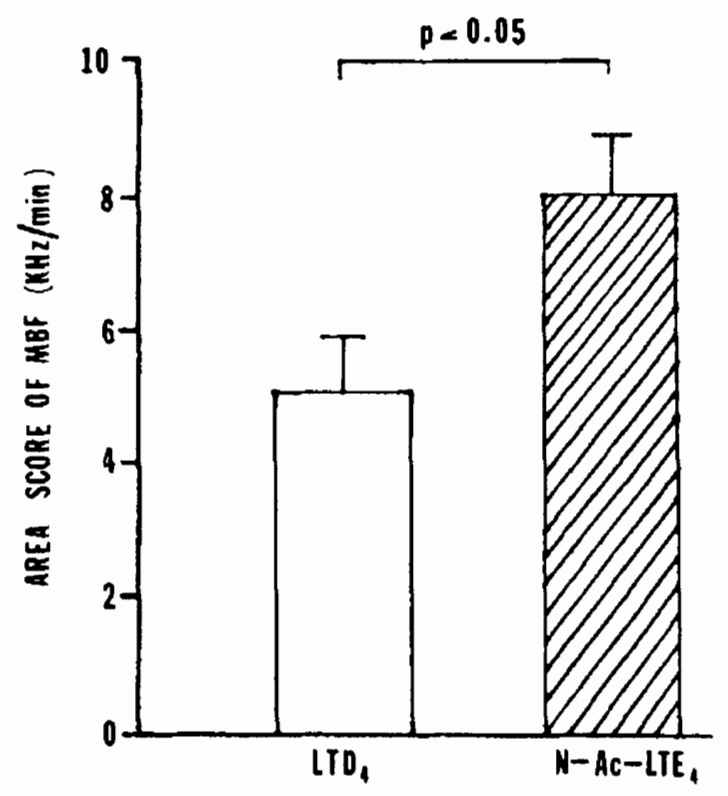

FIGURE 2. The areas score of the mesenteric blood flow response to $N$-Ac-LTE 4 . The overall effect of $N$-Ac- $\mathrm{LTE}_{4}$ is significantly larger than $\mathrm{LTD}_{4}$ at $p<0.05(n=6)$.

The effects of $N$-Ac-LTE 4 in the rat are substantially different from those described in the pig. ${ }^{3}$ The significant vasoconstrictor activity of $N$-Ac-LTE ${ }_{4}$ in the rat, taken together with this LT being the primary bile metabolite, and the possible enterohepatic recycling of LT suggest that $N$-Ac-LTE 4 might play an important role in systemic effects of LT in this species. Furthermore, the possible role of $N-A_{c}-L_{T E}$ in mediation of some of the overall LT effects also presents the need to evaluate LT antagonists against $N$-Ac-LTE 4 because the failure of some LT antagonists to effectively block $N$-Ac-LTE ${ }_{4}{ }^{3}$ might underlie their limited capacity to prevent or reverse $\mathrm{LT}$-mediated pathological processes.

\section{REFERENCES}

1. Denzlinger, C., S. RaAp, W. Hagmann \& D. KepPler. 1985. Leukotrienes as mediators in tissue trauma. Science 230: $330-332$. 
2. Denzlinger, C., A. Guhlman, W. Hagmann, P. H. Scheuber, F. Scheyerl \& D. KEPPLER. 1986. Cysteinyl leukotrienes undergo enterohepatic circulation. Prostaglandin Leukotriene Med. 21: 321-322.

3. Foster, A., B. Fitzsimmons \& G. LeTts. 1986. The synthesis of $N$-acetyl-leukotriene $\mathrm{E}_{4}$ and its effects on cardiovascular and respiratory function of the anesthetized pig. Prostaglandins 31: 1077-1086.

4. EimerL, J., A-L. Siren \& G. Feuerstein. 1986. Systemic and regional hemodynamic effects of leukotriene $D_{4}$ and $E_{4}$ in the conscious rat. Am. J. Physiol. 251: H700-H709. 